\title{
Changes in the topology of a concrete porous space in interactions with the external medium
}

\author{
Yury Mikhaylovich Bazhenova ${ }^{a^{*}}$, Vladimir Trofimovich Erofeev ${ }^{a}$, Vladimir Ivanovich Rimshin ${ }^{\mathrm{b}}$, \\ Sergey Vitalyevich Markov ${ }^{b}$ and Vladimir Leonidovich Kurbatov
}

\begin{tabular}{l}
\hline Article history: \\
Received 6 March, 2016 \\
Accepted 27 May 2016 \\
Available online \\
28 May 2016 \\
\hline Keywords: \\
Corrosion \\
Concrete \\
Capillary \\
Carbonization time \\
Mathematical model
\end{tabular}

${ }^{a}$ Mordovian N.P. Ogariov State University, Saransk, Russia

${ }^{b}$ Moscow State Construction University, Yaroslavskoye Highway, 26, Moscow, 129337, Russia

${ }^{c}$ North Caucasian branch of the federal government's budget educational institution of higher professional education Belgorod State Technological University VG Shukhov Zheleznovodskaya str., 24, Mineralnye Vody, Stavropol Territory, 357202, Russia

\section{A B S T R A C T}

\section{Introduction}

Concrete and reinforced concrete, since the 2-nd half of the 20-th century, have been basic construction materials satisfying demands of building industries. Results of natural explorations and expert evaluations show that over $50 \%$ of all the building structures, depending on the designation of buildings and houses, are subjected to the process of carbonization in the atmospheric medium. The influence of aggressive media goes together with a decrease in the lifetime of these structures, and with * Corresponding author.

E-mail addresses: 732687@mail.ru (Y. M. Bazhenov)

(C) 2016 Growing Science Ltd. All rights reserved. doi: $10.5267 /$ j.esm.2016.5.001

\begin{abstract}
The article is devoted to some aspects of research of hostile environment influence on the concrete durability. The mechanism of formation of chaotically diversified difficult structure space are examined. Measures for receiving conditions under which the volume of products of reaction will be equal to the volume of the reacted alkaline components of a cement stone are offered. High-quality and quantitative changes in a pore space, which are irrespective of density and the relative molecular mass of reaction products are described. Besides, movement and distribution of gaseous products of reaction in volumes of a cement stone are simulated. Reasonable integrated dependences of influence of aggressive environment on various types of concrete surfaces are given. The methods of corrosion control offered in article allow substantially to lower or absolutely to exclude adverse effect of hostile environment on concrete mix. In this case service life of concrete and reinforced concrete structures will increase.
\end{abstract}


losses arising from a stoppage of the production process for making repairs and reconstructions. In developed countries, the overall detriment due to corrosion of building constructions amounts to $4 \%$ of the national output and this detriment goes on increasing. The strength and durability of concrete in aggressive media are determined by the concrete structure which is formed in the process of cement hydration, the structure being of a capillary - porous character. Pores and capillaries having the dimension of $10^{-2}-10^{-7} \mathrm{~cm}$ form a chaotically diversified complex system (Alekseev, 1993; Bazhenov, 2002). Chemical interactions of cement hydration products with active components of diffusing external media occur inside the porous space.

Meland (1985) experimentally showed that the action of gaseous carbon dioxide results in changes of reciprocal proportions in pores of different dimensions. The volume of pores larger than $5 \mathrm{~nm}$ increases, whereas the volume of pores within the interval of 5-0.04 nm decreases (Neville, 1995). Carbonization changes the porous structure of hydrated cement pastes (Cebeci, 1986, OsherovMarshak, 2002). Since calcium hydrates contributes to formation of pores, the total porosity and volume concentration of large pores of the dimensions of 12.5-100 nm usually decrease (Dvorkin \& Dvorkin 2007 ) and the interaction process of concrete with an external aggressive medium starts on the concrete surface. As the front of the reaction moves off the surface, the external medium transfer into the reaction zone takes place via a corroded layer of the concrete, the diffusion coefficient of which will be different from the diffusion coefficient of a non-corroded layer of concrete. However, while elaborating mathematical models, some researchers including the members of the European Bureau for Concretes (CEB) (e.g. Meier et al., 2007; Orchard et al., 1979), do not take into account this physical change and uphold all physical parameters entering the equation of diffusion that are characteristic of the state of a non-corroded concrete. But it does not correspond with reality. With an aim to bridge mathematical models and real processes of corrosion, the Mindess (2006) resort to a complication of these models by introducing additional integers in the equation of corrosion, which, to a greater degree, complicates obtainable solutions and, at the same time, does not reflect a real physical state of a corroded zone of concrete. One of the main objectives at this stage is development of effective methods on an exception of this kind of destructions. Processes occurring in concrete - aggressive medium interactions and possible ways of elongating the lifetime of reinforced concrete structures are discussed in the present article.

\section{Theoretical framework}

An original approach to solving the problem of capillary integral volume transformations and capillary differential distributions over their dimensions in chemical interactions of concrete with the external medium is suggested as a result of the present investigation. This approach is thought to define, more correctly, the physical state of a corroded zone of concrete in the equation of diffusion and to describe more correctly, from a physical viewpoint, the transportation of the external medium to a reaction zone.

Let us introduce a new notion "internal specific concrete surface reactive capacity" and designate it as LS. This capacity is a ratio of volume density of products formed as a result of concrete reactions with the external aggressive medium $\mathrm{LV}\left(\mathrm{kg} / \mathrm{m}^{3}\right)$ to the area of the porous space surface per unit of the volume, $\mathrm{S}\left(\mathrm{m}^{2} / \mathrm{m}^{3}\right)$, i.e.

$$
L_{S}=\frac{L_{V}}{S}
$$

The volume density of reaction products is determined by the expressions (Bazhenov, 2002; Wu, 2006):

$$
L_{V}=C \times p \times f \times k
$$


where $\mathrm{C}$ is the cement consumption in concrete in $\mathrm{kg} / \mathrm{m} 3 ; p$ is the relative content of cement stone alkaline components involved in the reaction with aggressive medium ions, in portions of the unit; $f$ is the degree of completeness of the reaction of these interactions, it portions of the unit; $k$ is the concrete reactive capacity which is the content of an active bound component of the aggressive medium, in portions of the cement mass. From Eq. (1) it is seen that the concrete specific surface capacity has a dimensionally of $\mathrm{kg} / \mathrm{m}^{3}$ and shows a mass of reaction produced products per unit of a porous space surface area.

\subsection{The studied assumption}

Suppose that the concrete is an isotropic material with respect to the concrete reactive capacity, i.e. value $k$ is independent of the point position considered and is a feature of the structure of concrete as a quasi-homogeneous material. It is known that pores, in pore volumes per unit of a concrete volume and in their differential distributions over their dimensions in the volume unit, are quasihomogeneously distributed in the concrete volume. The thickness of adsorption tightly-bound moisture on the inner surface of pores depends on a sort of the liquid adsorbed and on the kind of the sorbent, but it does not depend on capillary dimensions, if their diameter exceeds the doubled thickness of adsorbed moisture molecules and properties of liquid thin layers, as a sort of wedging pressure, are not decisive. In these conditions, the specific surface concrete reactive, capacity Ls will not depend on pore dimensions characterized by the radius. It will depend only on concrete reactive properties and the aggressive medium (Kosuhin et al., 2013; Krasovskii, 2013; Kurbatov et al., 2010; Lomachenko, 2014).

Thus, the mass of reaction products from the reaction between cement stone alkaline components and aggressive medium active components, per unit of the porous space surface area, will be the same for small capillaries and for large pores, i.e. the equalities are valid:

$L_{S}\left(r_{i}\right)=$ const,$\frac{d L_{S}\left(r_{i}\right)}{d r_{i}}=0$.

At the known volume pore content $\mathrm{Vn}$ and inner surface area of all pores $\mathrm{S}$ per unit of the concrete volume, the pore hydraulic radius $r_{G}$ will be equal to the ratio $r_{G}=\frac{V_{n}}{S}$. Knowing the hydraulic radius, one may find the inner surface area SG of the unit of the pore length and the cross-section area AC. They will be equal: $\mathrm{S}_{\mathrm{G}}=2 \mathrm{nrG} ; \mathrm{A}_{\mathrm{G}}=\pi r_{G}^{2}$. Let us take into account that pore inner surfaces characterized by the dimension which is equal to the hydraulic radius $r_{G}$ possess the same properties as physical pores of the radius $r_{\mathrm{i}}$. On this basis, one may find the mass $\mathrm{mg}(\mathrm{kg})$ of products formed in reactions of cement stone alkaline components with an active acid medium component in a unit-length pore with a dimension equal to the hydraulic radius $r_{\mathrm{G}}$. This mass will be equal to:

$$
m_{G}=L_{S} \times S_{G}
$$

The mass, at the reaction product density which is equal to $\rho_{G}$, will take the volume in a unitlength pore:

$$
V_{G}=\frac{m_{G}}{\rho_{g}}=\frac{L_{S} \times S_{G}}{\rho_{G}} .
$$

The mass of the cement reactive alkaline component, for example $\mathrm{CaO}$, is determined, depending on the mass of produced products, from the equation of the interaction reaction with account for an equivalent radio. For example, for the reaction of carbonization this equivalent ratio is equal to:

$$
\frac{\mathrm{CaO}}{\mathrm{CaCO}_{3}}=\frac{56}{100}
$$


With this account, the reacted $\mathrm{CaO}$ mass will be equal to: $m_{G, \mathrm{CaO}}=m_{G} \frac{56}{100}$

\subsection{Equivalent coefficient}

At the known mass and density $\rho_{\mathrm{CaO}}$, the volume of the reacted $\mathrm{CaO}$ with account for the equivalent ratio will be determined as: $V_{G, \mathrm{CaO}}=\frac{m_{G, \mathrm{CaO}}}{\rho_{\mathrm{CaO}}}$.

The volume of accumulating products $\Delta \mathrm{V}_{\mathrm{G}}$ in a unit-length pore, with account for a loss of the volume taken by the reacted $\mathrm{CaO}$ in its equivalent ratio, is determined as the difference:

$$
\Delta V_{G}=V_{G}-V_{G, \mathrm{CaO}}=\frac{L_{S} \times S_{G}}{\rho_{G}}\left(1-\frac{\rho_{G}}{\rho_{\mathrm{CaO}}} \times \frac{56}{100}\right)
$$

When accumulating, this volume will gradually fill the pore volume, starting from the pore wall and approaching the center. Designating the thickness of the accumulating products as $\delta_{\mathrm{G}}$, we may write the expression for the volume $\Delta \mathrm{V}$ though $\delta \mathrm{G}$ and $r_{\mathrm{G}}$ on part of the pore length which is equal to the unit. This volume will be equal to:

$$
\Delta V_{G}=\pi r_{G}^{2}-\pi\left(r_{G}-\delta_{G}\right)^{2} .
$$

Substituting (DELTA)VG and SG by their values, the equality above will take the form:

$$
\delta_{G}=r_{G}-\sqrt{r_{G}^{2}-B r_{G}},
$$

where

$$
B=\frac{2 L_{s}}{\rho_{G}}\left(1-\frac{\rho_{G}}{\rho_{\text {CaO }}} \times \frac{56}{100}\right) .
$$

At $\delta \mathrm{G}=\mathrm{r}_{\mathrm{G}}$, the reaction products will fill in the volume of the pore of this radius. Substituting this value in Eq. (8), one may determine the radius at which capillaries and pores will be totally filled in by the reaction products:

$$
r_{c r}=B=\frac{2 L_{s}}{\rho_{G}}\left(1-\frac{\rho_{G}}{\rho_{C a O}} \times \frac{56}{100}\right) .
$$

All the capillaries and pores, whose radius $r<r_{\mathrm{cr}}$, will totally be filled in by the reaction products. On walls of the capillaries and pores, whose radius $r>r_{\mathrm{cr}}$, there will deposit reaction products of the thickness $\delta \mathrm{G}$. Let us re-write the expression in the brackets of Eq. (10) as

$$
r_{c r}=\frac{2 L_{S}}{\rho_{G}}\left(1-\frac{\rho_{G} \times G_{C a O}}{\rho_{C a O} \times G_{G}}\right),
$$

where $\mathrm{G}_{\mathrm{CaO}}$ is the relative molecular mass of cement $\mathrm{CaO}$ which is in the equivalent ratio with the reaction products formed in cement stone interactions with the external medium active component; $\mathrm{G}_{\mathrm{G}}$ is a relative molecular mass of the reaction products formed in the equivalent ratio with the cement $\mathrm{CaO}$ involved into the reaction.

\section{Discussion of the Results}

The analysis of Eq. (11) shows that when the expression in the brackets is equal to zero, the volume of reacted cement $\mathrm{CaO}$ will be equal to the volume of products formed in the reaction. If the reaction products are insoluble or little soluble, then they take, in the equivalent ratio, a free volume previously taken by $\mathrm{CaO}$. There should be no changes in a general picture of pore and capillary locations, either in the porous space integral volume or in the differential distribution of pores over dimensions (Novichkov, 2001). When the relative molecular mass of reaction products $\mathrm{G}_{\mathrm{G}}$ exceeds the relative molecular mass of $\mathrm{G}_{\mathrm{CaO}}$ at relative equalities of their densities, i.e. at $\rho_{\mathrm{G}}=\rho_{\mathrm{CaO}}$, the denominator in Eq. 
(11) in the brackets will be larger than the numerator. This is the evidence that the reaction product volume exceeds the reacted $\mathrm{CaO}$ volume taken by $\mathrm{CaO}$ before the reaction in their equivalent ratio. When insoluble or little solube, the reaction products will be accumulating in pores and in concrete capillaries, filling in capillaries of a small radius and producing a layer of insoluble compositions of thickness (delta) on the walls of capillaries and pores of large dimensions (Sagneva et al., 2012). Here at both integral volume of pores and differential picture of pore distributions over dimensions will change. The larger is the difference in the expression $\left(\rho_{\mathrm{CaO}} \times G_{G}-\rho_{G} \times G_{\mathrm{CaO}}\right)$, the larger is the difference between volumes of reaction products and volume of reacted $\mathrm{CaO}$ in their equivalent ratio.

\subsection{Statement of facts}

Therefore, a larger volume of pores will be taken by reaction products accumulating on their walls. If the volume of reaction products exceeds a permissible limit for this sort of concrete, then a pressure will appear on pore walls in the process of reaction product crystallization out of the mortar. When the value of stretching deformations in a cement stone around the pores due to this pressure exceeds the limit, there will appear, first, micro-crakes in the cement stone. Later, such cracks merge with the formation of entire cracks, resulting, finally, in scales of the worked-out part of the concrete crosssection. This argumentation is confirmed by the experimental investigations of carbonization (Solomatov \& Tahir, 1989; Stol nikov, 1953; Wu, 2006; Ramachandran et al., 1981). If the inequality $\rho_{G} \times G_{\mathrm{CaO}}>\rho_{\mathrm{CaO}} \times G_{G}$, is fulfilled, the relative molecular mass of the reaction products GG will be less than the molecular mass of the cement $\mathrm{CaO}$.

At a relative equality of their densities $\rho_{G}=\rho_{C a O}$, the volume of reaction products will be less than the volume taken by $\mathrm{CaO}$ before the reaction, in their equivalent ratio. In the case of insolubility or little solubility of the reaction products, there will take place the softening of the cement stone with an increase in the integral volume of a porous space and with a displacement of the pore distribution curve apex of pore differential distributions over their dimensions in the direction of an increase in the dimension of a pore average statistical radius (Dobrolyubov et al., 1983). The softening of the concrete cement stone may occur not only due to a decrease in the volume taken by the reaction products with respect to the volume of the reacted cement $\mathrm{CaO}$ in their equivalent ratio, but also due to the formation of reaction products soluble in liquid or gas and the formation of solutions or gaseous mixtures. In this case, the density $\rho_{G}$ of liquid or gaseous reaction products will be less than the density of $\rho_{\mathrm{CaO}}$ as a solid body, i.e. the inequality $\rho_{G}<\rho_{C a O}$ is observed. At the equivalent equality of the reaction products

and the reacted $\mathrm{CaO}$, (i.e. if the equality $G_{G}=G_{\mathrm{CaO}}$ is observed), then the expression in the brackets of Eq. (11) will take the form

$$
r_{c r}=2 L_{S}\left(\frac{1}{\rho_{G}}-\frac{1}{\rho_{C a O}}\right) \text {. }
$$

As seen from Eq. (12), the greater is the difference in the expression $\rho_{C a O}-\rho_{G}$, the greater is the softening which will result in an increase of the integral porous volume and an increase in the pore hydraulic radius. If a relative molecular mass of gaseous products of the reaction is larger than a relative molecular mass of the reacted $\mathrm{CaO}$ in their equivalent ratio, then the inequality $\rho_{\mathrm{CaO}} \times G_{G}>\rho_{\mathrm{CaO}} \times G_{\mathrm{CaO}}$ is observed. This is the evidence that the volume of the liquid or gaseous reaction products will be larger than the volume of cement stone components. If the difference in these volumes exceeds the porous volume in which the exchange reaction has taken place, the liquid reaction products will flow off the concrete body as mucus. The migration of gaseous reaction products will take place independently of the difference in volumes of the reaction and cement stone components in their equivalent ratio, according to the mass transfer law, either by diffusion or by effusion. 
Qualitative and quantitative changes in a porous space, independently of the density and relative molecular mass of the reaction products, will be determined to a great extent by the specific surface concrete reactive capacity LS. With an increase in LS, volume of the reaction products will increase, which, in case of insolubility or weak solubility of reaction products, will lead to a decrease in the porous space integral volume and to a shift of the curve apex of the differential expansion of pore dimensions in the direction of a decrease in the pore radius (Furmanov, 2009; Ureckaya \& Batyanowski, 2001; Valsov, 1998; Liang \& Lin, 2003; Papadakis \& Efstathion, 2006).

\section{Conclusions}

For the first time the analytical expression for a critical dimension of a capillary radius $r_{\mathrm{cr}}$ for a cement stone of concretes affected by an aggressive external medium has been obtained. The capillary radius $r_{\text {cr }}$ dependence on the integral specific concrete surface reactive capacity LS was discovered. All capillaries of the radius $r<r_{\mathrm{cr}}$ will be filled in by products of the reaction between a diffusing external aggressive medium and reactive components of a cement stone. If the reaction products are insoluble or little soluble, they will create, when accumulating, a pressure on walls of capillaries of given dimensions and will destroy the concrete integrity in the forms of cracks and scales in the concrete.

In known conditions of exploitation characterized by a chemical composition of the external medium, one can avoid the above-mentioned damage as early as at the stage of selecting concrete compositions by regulating the value of the internal specific concrete surface reactive capacity LS. This can be made by the introduction into the concrete of a mixture of special chemical additives contributing to a decrease of a relative molecular mass of reaction products in comparison with a relative molecular mass of reacted alkaline components of the cement stone. In this case, it is desirable to obtain the conditions that the volume of the reaction products be equal to the volume of the reacted alkaline components of the cement stone or should exceed it insignificantly. In this case, the density of the concrete in the process of its exploitation will increase with no damage in its structure, thus making a barrier to the diffusion of the external medium enhancing the lifetime of concrete and reinforced concrete frames.

\section{Acknowledgements}

The authors are thankful to Mr. A. Ouzoykin and Mrs. A. Picksaikina for preparing the manuscript for publication.

\section{References}

Alekseev, S. N. (1993). Durability of reinforced concrete in aggressive media (Vol. 96). AA Balkema Publishers.

Bazhenov, Y.M. (2002). Concrete topology Manual. Moscow: ACB.

Cebeci, O.Z. (1986). Hydration and porosity of cement paste cured in warm and dry environments, $8^{\text {th }}$ International Congress on the on the Chemistry of Cement, Sept. 22-27, Rio de Janeiro, Brasil, vol. 3 (2.2), $412-416$

Dobrolyubov, G., Ratinov, V.B., \& Rosenberg, T.I. (1983). The prediction of durability of concrete. Moscow.

Dvorkin, L. I., \& Dvorkin, O. L. (2007). Building materials from waste the Industrial Training handbook Rostov n/D: Phoenix,-368 p. Building. [in Russian].

Lomachenko, D.V. (2014). Rheological properties research of cement paste with superplasticizing agent for concrete SB-3. Modern Science-Incentive Technologies, 2, 123.

Furmanov, N.E. (2009). Favorable composition of the concrete for the manufacture of waterproof structures according to the "White tub". Civil Engineering Journal, 3, 11-16. 
Kosuhin M.M., Poluektova V.A., Malinovka V.M., \& Shapovalov N.A. (2013). Polyfunctional super plasticizer for concretes based on pyrocatechin production waste products. Fundamental Research, 3(1), 718-722.

Krasovskii P.F. (2013). Physical-chemical principles of formation of structure of cement concretes. Khabarovsk: Publishing house FESTU.

Kurbatov V.I., Sereda O.A., \& Danilyan E.A. (2010). The dilatometric method of testing concrete. Diagnostics of materials, 6; 61-63.

Liang, M. T., \& Lin, S. M. (2003). Mathematical modeling and applications for concrete carbonation. Journal of Marine Science and Technology, 11(1), 20-33.

Meland, I. (1985). Carbonation effects in hardened fly ash cements. In MRS Proceedings (Vol. 65, p. 199). Cambridge University Press.

Meier, S. A., Peter, M. A., Muntean, A., \& Böhm, M. (2007). Dynamics of the internal reaction layer arising during carbonation of concrete. Chemical Engineering Science, 62(4), 1125-1137.

Mindess, S. (2006). Advanced concrete for use in civil engineering. Advanced Civil Infrastructure Materials, 1-29.

Neville, A. M. (1995). Properties of concrete.

Novichkov, P.I. (2001). On diffusion kinetics of reactions in concrete. Proceedings of the Jubilee Conference, Moscow: PAACH.

Orchard, D. F., Curran, A., \& Hearne, R. (1979). Concrete Technology: Properties of Materials. 1, (No. Monograph).

Osherov - Marshak, A. (2002). Methodological aspects of modern concrete technology. Concrete and Reinforced concrete, 11, 5-7.

Papadakis, V. G., \& Efstathiou, M. P. (2006) Field Validation of a Computer-based Prediction for Concrete Service Life.

Sagneva E.A., Martakov I., Dmitrienko R.A. \& Dmitrienko C. (2012). Analysis of the state of mixing equipment for concrete and mortar mixes. Energy-saving technological systems and equipment for manufacture of building materials: interuniversity collection of articles, 11, 315-317.

Solomatov V.I., \& Tahir M.K. (1989). Tucker Shah Md. The intensive technology of concrete, Moscow: Stroiizdat.

Stol'nikov, V.V. (1953). Air entraining additives in hydraulic concrete. Leningrad: Gosenergoizdat.

Ramachandran, V. S., Feldman, R. F., \& Beaudoin, J. J. (1981). Concrete Science: Treatise on Current Research, Heyden and Son. Ltd., Philadelphia.

Ureckaya, E.A., \& Batyanowski, E.I. (2001). Dry mix: materials and technologies. Minsk; Strinko.

Vlasov, V.K. (1998). Regularities optimization of structure of concrete with dis - dispersed mineral additives. Concrete and Reinforced Concrete, 4, 10-12.

Wu, S.H. (2006). Advanced cement composites for use in civil engineering. Science, Mechanics and Applications, 63-117. 